\title{
Síntese e caracterização de ferritas de Zn e Mn provenientes de pilhas inutilizadas
}

\section{(Synthesis and characterization of Zn and Mn ferrites from spent batteries)}

\author{
M. R. Baldissera, M. R. A da Silva, C. A. Silveira, R. M. Lima, S. A. Maia, M. R. da Silva, D. A. W. Soares, R. Gimenes \\ Universidade Federal de Itajubá-UNIFEI, Instituto de Física e Química (IFQ) \\ Av. BPS 1303, Itajubá, MG 37500-903 \\ mbaldissera@unifei.edu.br
}

\begin{abstract}
Resumo
Atualmente há uma crescente demanda no cenário mundial com relação ao tratamento de resíduos industriais, dentre estes o fenol e pilhas inutilizadas. Óxidos e cerâmicas a base de ferro, tais como ferritas são utilizadas como catalisadores no processo Fenton heterogêneo. Os processos de Fenton e foto-Fenton apresentam alta eficiência no tratamento de resíduos tóxicos utilizando catalisadores a base de ferro. Neste contexto foram desenvolvidas ferritas a partir de resíduos de pilhas inutilizadas. O uso destas ferritas como catalisadores é bastante interessante, uma vez que, são materiais ferromagnéticos podendo ser recuperados no final do processo utilizando campo magnético, evitando a liberação de $\mathrm{Fe}^{2+}$ e $\mathrm{Fe}^{3+}$ no efluente. Os resultados mostraram que a ferrita proveniente de pilhas inutilizadas e calcinada à temperatura de $500{ }^{\circ} \mathrm{C}$ apresentou estrutura correspondente a fase espinélio e magnéticas $\left(\mathrm{M}_{\mathrm{S}}=37,04\right.$ emu. $\left.\mathrm{g}^{-1}\right)$ e como catalisador de fotodegradação atingiu-se $90 \%$ de remoção de fenol em pH 2,5 durante $2 \mathrm{~h}$ de irradiação.

Palavras-chave: ferritas, material magnético, pilhas, foto-Fenton.
\end{abstract}

\begin{abstract}
Currently there is a growing global demand with respect to industrial waste treatment, among them phenol and spent batteries. Iron oxides and ceramics containing iron, such as ferrites, have been used with success as catalysts for heterogeneous Fenton process. The Fenton and photo-Fenton processes have high efficiency in treatment of toxic residue using iron as catalysts. In this respect, ferrites were developed from spent batteries. The use of these ferrites as catalysts is very interesting because these oxides are ferromagnetic and can be recovered at the end of the process using a magnetic field, avoiding the discharge of $\mathrm{Fe}^{2+}$ and $\mathrm{Fe}^{3+}$ in the environment. The results showed that the ferrite from spent batteries calcined at $500{ }^{\circ} \mathrm{C}$ presents structural of spinel phase and magnetic properties $\left(M s=37.04\right.$ emu. $\left.g^{-1}\right)$ and applied in photo-Fenton process, it was reached 90\% of phenol removal at pH 2.5 with 2 h of irradiation.
\end{abstract}

Keywords: ferrites, magnetic material, spent batteries, photo-Fenton.

\section{INTRODUÇÃO}

As pilhas e baterias estão presentes no nosso cotidiano em produtos eletrônicos, controles remoto, celulares, entre outros aparelhos eletroeletrônicos, gerando um grande consumo deste tipo de material. No entanto, o seu descarte ainda não é adequado, sendo que maior parte das pilhas produzidas ainda são descartadas no lixo urbano e encaminhadas aos aterros sanitários, contaminando solo e lençóis freáticos. Algumas pilhas ainda são compostas de elementos químicos tóxicos como cádmio, mercúrio e chumbo, altamente prejudiciais a fauna e a flora. Dentre os compostos presentes nas pilhas também estão o ferro, manganês e zinco, os quais podem ser utilizados na obtenção de materiais magnéticos [1]. Desta forma, a reciclagem das pilhas inutilizadas apresenta-se como uma solução ambiental, além de proporcionar o desenvolvimento de novos materiais a partir de seus compostos químicos.

O intuito deste trabalho é desenvolver um produto capaz de agregar valor as pilhas descartadas, empregando um novo produto gerado a partir dos resíduos de pilha na degradação de substâncias nocivas, tais como compostos fenólicos. O fenol é um resíduo tóxico bastante problemático com relação a sua degradação. No Brasil, $64 \mathrm{~kg}$ de fenol são lançados na atmosfera, provenientes de termoelétricas movidas à carvão. Além disso, efluentes indústrias, hospitalares e domésticos também podem conter o composto e com isso contaminar as águas. Por ser uma substância com altos riscos à saúde e por possuir elevado potencial de contaminação ambiental [2], faz-se necessário o desenvolvimento de processos que facilitem a remoção deste composto do ambiente. Uma possibilidade de mineralização do fenol é o emprego de 
processos oxidativos avançados (POA). Estes processos baseiam-se na geração do radical hidroxila como o principal agente oxidante [3]. O processo de Fenton, um dos principais POA, utiliza catalisadores à base de ferro, que reagem com o peróxido de hidrogênio (adicionado ao sistema) liberando o radical hidroxila $\left(\bullet^{\circ} \mathrm{OH}\right)[3,4]$. A incidência de radiação no sistema de tratamento por Fenton, denominado processo fotoFenton promove a foto-redução de $\mathrm{Fe}^{3+}$ a $\mathrm{Fe}^{2+}$, aumentando a eficiência na geração de radicais hidroxila [5]. A aplicação do processo Fenton/foto-Fenton para o tratamento de resíduos industriais e agrícolas já é bem conhecida [6-9]. $\mathrm{O}$ processo foto-Fenton, é limitado por uma estreita faixa de $\mathrm{pH}(2,5-3,0)$, em valores acima dessa faixa o $\mathrm{Fe}(\mathrm{III})$ precipita e em valores abaixo de 2,5 altas concentrações de $\mathrm{H}^{+}$podem sequestrar radicais hidroxila. Uma alternativa para solucionar esse problema, é a utilização de complexo de ferro ou ferro imobilizado, o que também é conhecido como processo heterogêneo de Fenton [7, 10].

Óxidos de ferro e cerâmicas contendo ferro tais como ferritas, têm sido empregados com sucesso como catalisadores no processo heterogêneo de Fenton. Nanopartículas de ferrita de cobalto, $\mathrm{CoFe}_{2} \mathrm{O}_{4}$ (diâmetro de $10 \mathrm{~nm}$ ) encapsuladas em partículas mesoporosas de carbono foram empregadas como reagente de Fenton, nesse estudo, a velocidade de degradação de $\mathrm{H}_{2} \mathrm{O}_{2}$ foi de $0,111 \mathrm{~min}^{-1}$, indicando a reatividade do sistema na geração de $\bullet \mathrm{OH}$ [11]. Outros três tipos de óxidos de ferro foram empregados como catalisadores de Fenton na degradação de Bisfenol A (BPA). Constatou-se que o emprego deste catalisador apresenta eficiência oxidativa superior ao processo de Fenton homogêneo [12].

O uso de ferritas como catalisadores no processo heterogêneo de Fenton é bastante interessante, pois além de possibilitar a ocorrência do processo de Fenton em maior faixa de $\mathrm{pH}$, a rota complexação-imobilização do ferro em estruturas micro ou mesoporosas permite a retenção do ferro utilizado no processo, tornando este processo viável para tratamento de grandes quantidades de resíduos, sem a necessidade de adequar o descarte dos íons Fe ao limite imposto pela legislação - $15 \mathrm{mg} / \mathrm{L}$ [13]. Uma vez que, estes óxidos são ferromagnéticos e sofrem interação com um campo magnético externo, o catalisador pode ser recuperado ao final do processo pela aplicação de um campo magnético, evitando a liberação de íons $\mathrm{Fe}^{2+}$ ou $\mathrm{Fe}^{3+}$ no efluente.

Neste trabalho foram sintetizadas ferritas de zinco e manganês utilizando pilhas descartadas como fonte de Fe, $\mathrm{Zn}$ e Mn. Os catalisadores desenvolvidos foram caracterizados por difração de raios X (DRX) e magnetômetro de amostra vibrante (VSM). A eficiência da ferrita como catalisador foi avaliada por experimentos de fotodegradação utilizando como composto-alvo o fenol.

\section{EXPERIMENTAL}

Primeiramente foi realizado o desmonte das pilhas, cujas partes foram separadas em carcaça, papel, plástico, eletrodo e pasta eletrolítica (PE). A carcaça metálica (fonte de Fe) foi dissolvida em ácido sulfúrico $\left(5 \mathrm{~mol} . \mathrm{L}^{-1}\right)$ e filtrada. A pasta eletrolítica (fonte de $\mathrm{Mn}$ e $\mathrm{Zn}$ ) foi calcinada a 400 ${ }^{\circ} \mathrm{C} / 4 \mathrm{~h}$ para a remoção de toda a matéria orgânica contida no material de partida, em seguida esta pasta foi adicionada a solução de ferro e mantida em ponto de ebulição por 2 h. A estequiometria da ferrita foi corrigida adicionando $\mathrm{ZnO}$ à solução considerando a concentração de $\mathrm{Zn}, \mathrm{Mn}$ e $\mathrm{Fe}$ determinados por fluorescência de raios X (Tabela I). Foi adicionado à esta solução ácido cítrico mantendo sob agitação a $60^{\circ} \mathrm{C}$ até a obtenção de uma solução clara e viscosa de metais complexados. Etilenoglicol foi adicionado para esterificar a solução a $80^{\circ} \mathrm{C} / 4 \mathrm{~h}$ até atingir alta viscosidade. Após este procedimento, o material foi calcinado a 400 ${ }^{\circ} \mathrm{C} / 4 \mathrm{~h}$, o material obtido foi moído e calcinado novamente nas seguintes temperaturas 500,800 e $1100{ }^{\circ} \mathrm{C} / 4 \mathrm{~h}$ para a obtenção do pó de ferrita.

A estrutura da ferrita foi investigada por difração de raios $\mathrm{X}$ (Rigaku, Rint 2000), radiação $\mathrm{Cu}-\mathrm{k}_{\alpha}(\lambda=1,5406$ A) com passo de $0,2^{\circ}$ e varredura em $2 \theta$ de 20 a $80^{\circ}$. As propriedades magnéticas foram determinadas em um magnetômetro de amostra vibrante (LakeShore 7410) em temperatura ambiente e campo magnético de $\pm 15 \mathrm{kG}$. Os experimentos de fotodegradação foram conduzidos em um foto-reator do tipo batelada $\left(\lambda_{\text {máx }}=365 \mathrm{~nm}\right)$ de escala laboratorial utilizando fenol $0,1 \mathrm{~g}$. $\mathrm{L}^{-1}$ como composto alvo de degradação o qual teve sua concentração determinada pelo método colorimétrico [14] modificado [15]. Foram realizados experimentos de fotodegradação com 0,1 g.L ${ }^{-1}$ de fenol, $0,1 \% \mathrm{~m} / \mathrm{v}$ de ferrita e $10 \mathrm{mmol} \cdot \mathrm{L}^{-1}$ de $\mathrm{H}_{2} \mathrm{O}_{2}$ por 2 $\mathrm{h}$ de irradiação. Todos os experimentos foram realizados em triplicata e os resultados são uma média dos experimentos.

\section{RESULTADOS E DISCUSSÃO}

A Tabela I apresenta a porcentagem dos principais elementos presentes na pasta eletrolítica antes e após a calcinação a $400{ }^{\circ} \mathrm{C} / 4 \mathrm{~h}$. Estes resultados foram obtidos por meio de análise de fluorescência de raios X. Nota-se que os metais contidos na pasta estão sob a forma de óxidos e cloretos, sendo os principais componentes: manganês (37\%), zinco (19\%) e íons cloreto (18\%). Os dados obtidos para a concentração de Mn e Zn são similares aos apresentados [1] assegurando a composição da pasta e permitindo um melhor controle estequiométrico na mistura para a obtenção do material magnético na estequiometria desejada, para formar a fase espinélio de $\mathrm{ZnFeO}_{4}$ e $\mathrm{MnFeO}_{4}$. Era esperado que houvesse aumento da concentração de íons $\mathrm{Zn}$, já que após tratamento térmico a $400^{\circ} \mathrm{C}$ a matéria orgânica foi eliminada, concentrando desta forma os metais não voláteis nesta temperatura, tal como Mn e Zn. No entanto a concentração de Zn diminui um pouco, estando dentro do erro experimental do instrumento que é $2,5 \%$, em função da precisão da técnica de fluorescência de raios X estar relacionada a alterações na matriz da amostra a qual variou ligeiramente após a calcinação a $400{ }^{\circ} \mathrm{C}$. A concentração de ferro na ferrita aumentou neste material em relação à pasta eletrolítica devido à dissolução da carcaça de aço que foi usada como fonte de ferro. 
Tabela I - Porcentagem dos principais elementos contidos na pasta eletrolítica (PE) e ferrita. [Table I-Percentage of the main elements contained in the electrolytic paste (EP) and ferrite.]

\begin{tabular}{lcccccccc}
\hline & $\% \mathrm{Mn}$ & $\% \mathrm{Zn}$ & $\% \mathrm{Fe}$ & $\% \mathrm{Al}$ & $\% \mathrm{Cl}$ & $\% \mathrm{Na}$ & $\% \mathrm{Si}$ & $\% \mathrm{~K}$ \\
\hline PE temperatura ambiente & 37,32 & 19,52 & 2,36 & 7,14 & 18,3 & 0,001 & 0,61 & 1,17 \\
PE após $400{ }^{\circ} \mathrm{C} / 4 \mathrm{~h}$ & 43,91 & 18,15 & 3,08 & 4,62 & 1,19 & 3,09 & 1,52 & 0,94 \\
Ferrita & 10,14 & 3,67 & 55,6 & 0,66 & 0,11 & 0,04 & 0,31 & 0,15 \\
\hline
\end{tabular}

Erro instrumental 2,5\%

A análise da estrutura cristalina das ferritas foi feita por difração de raios $\mathrm{X}$; os resultados revelaram a formação da fase tipo espinélio (Fig. 1), semelhante a $\mathrm{MnFe}_{2} \mathrm{O}_{4}$ [11] e $\mathrm{ZnFe}_{2} \mathrm{O}_{4}$ [16], com estrutura cúbica e grupo espacial Fd$3 \mathrm{~m}$, indexada pelo arquivo JCPDS 74-2399. Para todas as temperaturas de calcinação houve a formação do espinélio de $\mathrm{Mn}_{\mathrm{x}} \mathrm{Zn}_{\mathrm{y}} \mathrm{Fe}_{2} \mathrm{O}_{4}(\mathrm{x}+\mathrm{y}=1)$, no entanto, com o aumento da temperatura de calcinação, acima de $800{ }^{\circ} \mathrm{C}$, notam-se picos referentes à fase contaminante $\alpha-\mathrm{Fe}_{2} \mathrm{O}_{3}$, com estrutura romboédrica e grupo espacial $\mathrm{R}-3 \mathrm{c}$ indexada pelo arquivo JCPDS 72-469. A influência do aumento da temperatura de calcinação na composição das fases no material sintetizado pode ser explicada pela natureza dos íons metálicos presentes, os quais são complexados pelo ácido cítrico. A esterificação do ácido cítrico pela adição do etileno glicol permite a estabilização destes metais organizados estruturalmente. Com a calcinação do material a $500{ }^{\circ} \mathrm{C}$ a resina polimérica é degradada e o arranjo estrutural permite a estabilização da fase espinélio $\mathrm{Mn}_{\mathrm{x}} \mathrm{Zn}_{\mathrm{y}} \mathrm{Fe}_{2} \mathrm{O}_{4}$ sem a formação de fases intermediárias. A intensidade da radiação de fundo observada no difratograma da Fig. 3a sugere que a ferrita obtida é cristalina. Nos difratogramas apresentados nas Figs. $3 \mathrm{~b}$ e $3 \mathrm{c}$ observou-se que uma parte da fase espinélio de $\mathrm{Zn}$ Mn foi degradada, e ocorreu a formação da fase $\alpha-\mathrm{Fe}_{2} \mathrm{O}_{3}$, a qual apresenta picos de maior intensidade relativa com o aumento da temperatura de calcinação de 800 para $1100^{\circ} \mathrm{C}$. O aumento da temperatura de calcinação em atmosfera oxidante (atmosfera convencional) pode favorecer a oxidação dos íons $\mathrm{Mn}^{2+} \mathrm{e}$ conduzir a formação de $\mathrm{MnO}_{2}$. No entanto, não foram identificados picos referentes a reflexão dos planos cristalinos das fases $\mathrm{MnO}_{2}, \mathrm{ZnO}$ ou $\mathrm{ZnO}_{2}$. Desta forma a presença da fase $\alpha-\mathrm{Fe}_{2} \mathrm{O}_{3}$ pode ser explicada pela estabilidade relativa dos cátions $\mathrm{Fe}^{3+}$ nos sítios tetraédricos (A), bem como na influência da atmosfera no equilíbrio envolvendo gás oxigênio e íons oxigênio intersticiais. Este mecanismo foi proposto [17], no qual, ferritas calcinadas a $950{ }^{\circ} \mathrm{C}$ com o teor de Mn próximos ou maior que $0,2(\mathrm{x}+\mathrm{y}=1)$ estabilizam cátions $\mathrm{Mn}^{2+}$ nos sítios tetratédricos (A), enquanto que cátions $\mathrm{Mn}^{3+}$ formados em temperaturas superiores a $750{ }^{\circ} \mathrm{C}$ são estabilizados em sítios octaédricos (B), e íons $\mathrm{Fe}^{2+}$ são oxidados a $\mathrm{Fe}^{3+}$ absorvendo oxigênio atmosférico. Os íons $\mathrm{Fe}^{3+}$ são extremamente instáveis nas posições tetraédricas, e desta forma, a tendência é de estabilizar uma fase cristalina de $\alpha-\mathrm{Fe}_{2} \mathrm{O}_{3}$. Assim, para temperaturas de calcinação de $800^{\circ} \mathrm{C}$ ou superior em atmosfera oxidante ocorre a estabilização da fase de $\alpha-\mathrm{Fe}_{2} \mathrm{O}_{3}$ além da ferrita de $\mathrm{Zn}-\mathrm{Mn}$ (estrutura espinélio). Desta forma pode-se dizer que o aumento da temperatura de calcinação favoreceu a cristalização das fases presentes, pois se observa a diminuição da radiação de fundo, no entanto também ocorreu a formação da fase contaminante $\alpha-\mathrm{Fe}_{2} \mathrm{O}_{3}$, Assim, pode-se afirmar que a calcinação na temperatura de $500{ }^{\circ} \mathrm{C}$ é a temperatura ideal para a formação de ferritas de $\mathrm{Zn}-\mathrm{Mn}$.

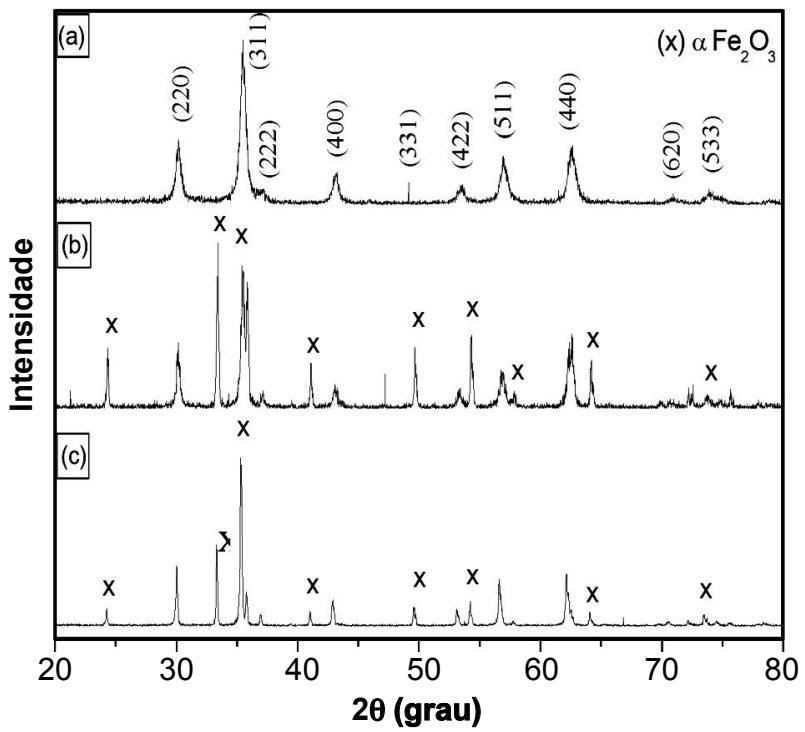

Figura 1: Difratogramas de raios $\mathrm{X}$ da ferrita obtida de pilha, calcinada a $500{ }^{\circ} \mathrm{C}$ (a), $800{ }^{\circ} \mathrm{C}(\mathrm{b}), 1100{ }^{\circ} \mathrm{C}$ (c).

[Figure 1: X-ray diffraction patterns of ferrite obtained from spent batteries, calcined at $500{ }^{\circ} \mathrm{C}($ a $\left.), 800^{\circ} \mathrm{C}(\mathrm{b}), 1100{ }^{\circ} \mathrm{C}(\mathrm{c}).\right]$

A caracterização magnética das ferritas foi realizada em um magnetômetro de amostra vibrante em temperatura ambiente; os resultados são apresentados na Fig. 2. A curva $\mathrm{M}-\mathrm{H}$ mostra que as ferritas comportam-se como material ferromagnético mole. A ferrita calcinada a $500{ }^{\circ} \mathrm{C}$ apresentou magnetização de saturação Ms de 37,04 emu. $\mathrm{g}^{-1}$. As demais ferritas calcinadas a temperaturas maiores apresentaram magnetização de saturação extremamente baixa, sendo $\mathrm{Ms}=$ 1,21 emu. $\mathrm{g}^{-1}$ para a ferrita calcinada a $800{ }^{\circ} \mathrm{C}$ e $\mathrm{Ms}=10,21$ emu.g ${ }^{-1}$ para a ferrita calcinada a $1100{ }^{\circ} \mathrm{C}$. Estes valores estão bem abaixo dos valores encontrados na literatura para ferritas de $\mathrm{Zn}-\mathrm{Mn}: 78,83$ emu.g ${ }^{-1}\left(\mathrm{Mn}_{0,26} \mathrm{Zn}_{0,24} \mathrm{FeO}_{2}\right)$ [18], 40,0 emu.g ${ }^{-1}\left(\mathrm{Ni}_{0,5} \mathrm{Zn}_{0,5} \mathrm{Fe}_{1,9} \mathrm{Cr}_{0,1} \mathrm{O}_{4}\right)$ [19], e 66 emu. $\mathrm{g}^{-1}$ $\left(\mathrm{ZnFe}_{2} \mathrm{O}_{4}\right)$ [16]. O comportamento apresentado pelas ferritas calcinadas em temperaturas elevadas pode ser explicado pela presença da fase antiferromagnética $\alpha-\mathrm{Fe}_{2} \mathrm{O}_{3}$, a qual devido ao acoplamento do momento magnético entre os átomos e 
os íons adjacentes, resulta em um alinhamento antiparalelo dos momentos magnéticos, ocasionando o cancelamento dos dipolos magnéticos.

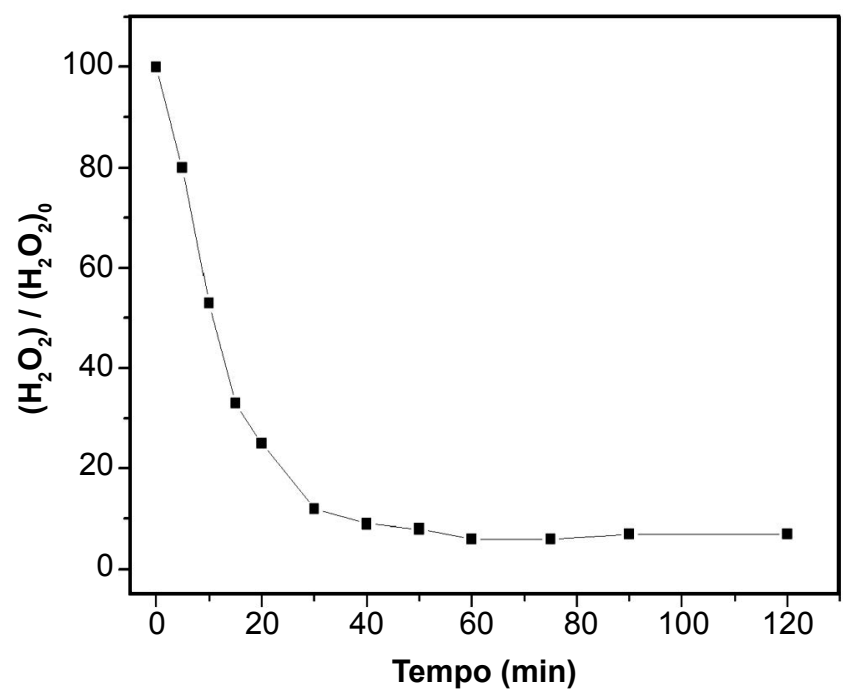

Figura 2: Curva de magnetização em função do campo magnético aplicado para as ferritas de pilha calcinadas em diferentes temperaturas.

[Figure 2: Magnetization curve as a function of the magnetic field obtained for ferrite of spent batteries calcined at different temperatures.]

Devido ao fato de a ferrita calcinada a $500{ }^{\circ} \mathrm{C}$ apresentar estrutura espinélio e melhor resultado de magnetização, esta foi empregada como catalisador no processo fotoFenton. Foram realizados experimentos para verificar a decomposição de $\mathrm{H}_{2} \mathrm{O}_{2}$ na presença de ferrita, os resultados mostraram que houve $94 \%$ de remoção de $\mathrm{H}_{2} \mathrm{O}_{2}$ (Fig. 3), confirmando a capacidade da ferrita em decompor o $\mathrm{H}_{2} \mathrm{O}_{2}$. Foi observado (Fig. 4) remoção próxima de $30 \%$ em $2 \mathrm{~h}$

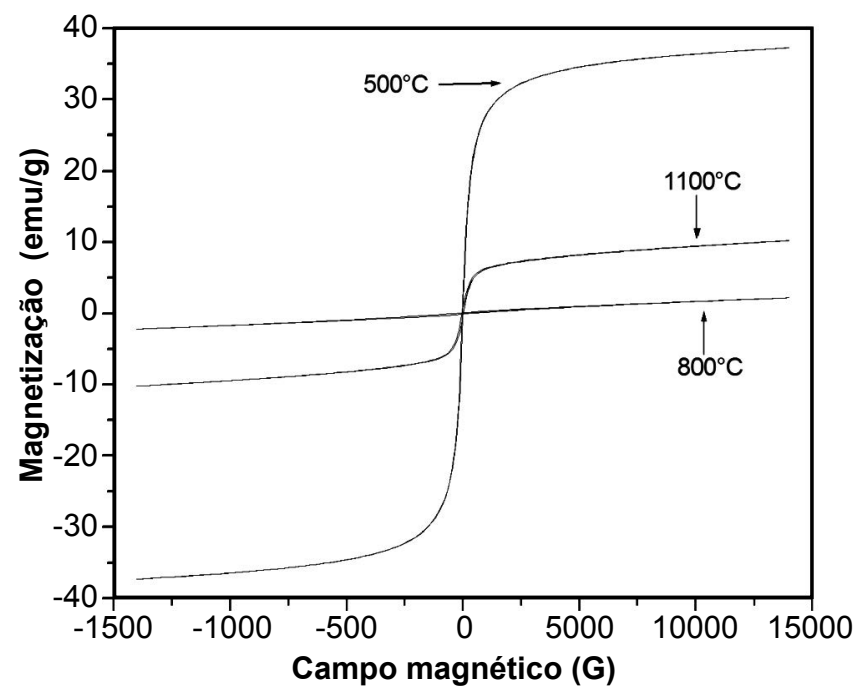

Figura 3: Decomposição de $\mathrm{H}_{2} \mathrm{O}_{2}$ na presença de ferrita. Condições: ferrita $(0,1 \% \mathrm{~m} / \mathrm{v}), \mathrm{H}_{2} \mathrm{O}_{2}(10 \mathrm{mmol} \mathrm{L}-1) ;(\lambda=365 \mathrm{~nm})$.

[Figure 3: Decomposition of $\mathrm{H}_{2} \mathrm{O}_{2}$ in the presence of ferrite. Ferrite $\left.(0.1 \% \mathrm{~m} / \mathrm{v}), \mathrm{H}_{2} \mathrm{O}_{2}\left(10 \mathrm{mmol} \mathrm{L} \mathrm{L}^{-1}\right) ;(\lambda=365 \mathrm{~nm}).\right]$ de irradiação em valor de $\mathrm{pH}$ de 5,5 . No entanto, quando o $\mathrm{pH}$ foi ajustado para 2,5, melhores resultados foram obtidos e observou-se uma remoção de aproximadamente $90 \%$ de fenol. Uma hipótese para explicar o aumento na remoção de fenol após ajustar o pH, é a possível liberação de íons de Fe da estrutura da ferrita através da solução, propiciando a ocorrência de Fenton homogêneo, aumentando a eficiência da reação. Porém, experimentos feitos para avaliar a estabilidade da ferrita não mostraram a presença de $\mathrm{Fe}^{2+}$ ou $\mathrm{Fe}^{3+}$ na solução, indicando que a reação de degradação de fenol está acontecendo na superfície da ferrita como proposto por [20]. Assim, nestas condições a ferrita é um catalisador com uma boa fotoatividade. Um fator muito importante no uso de ferritas como catalisador neste tipo de reação, é que ao final do processo o catalisador foi removido simplesmente com o auxílio de uma barra magnética.

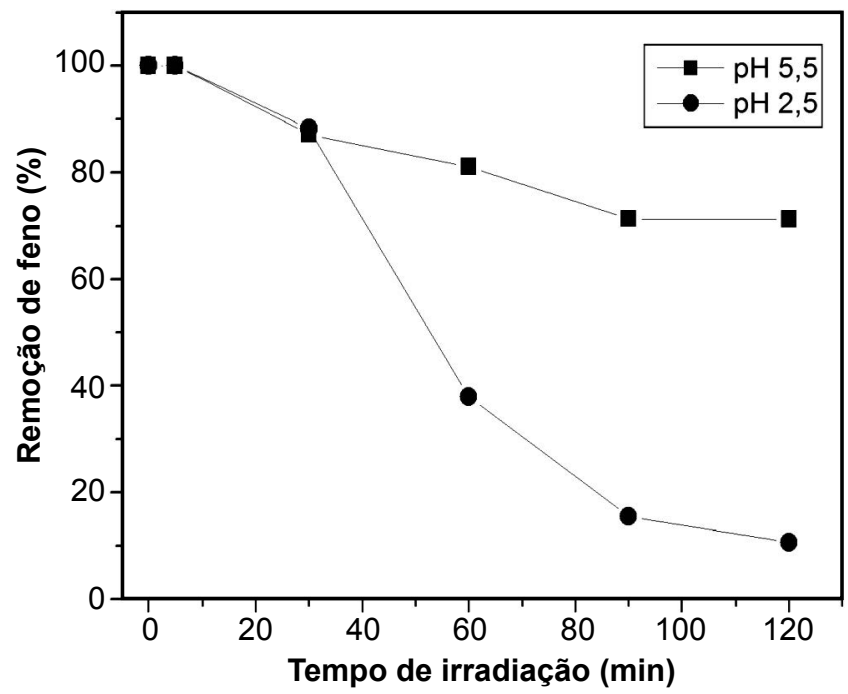

Figura 4: Remoção de fenol pelo processo foto-Fenton em pH 5,5 e 2,5. Condições: fenol 0,1 g. $\mathrm{L}^{-1}$, ferrita $0,1 \%(\mathrm{~m} / \mathrm{v}), \mathrm{H}_{2} \mathrm{O}_{2}(10$ mmol.L $\left.{ }^{-1}\right) ;\left(\lambda_{\max }=365 \mathrm{~nm}\right)$.

[Figure 4: Phenol removal by heterogeneous photo-Fenton process in $\mathrm{pH} 5.5$ and 2.5. Phenol $0.1 \mathrm{~g} . \mathrm{L}^{-1}$, ferrite $0.1 \%(\mathrm{~m} / \mathrm{v}), \mathrm{H}_{2} \mathrm{O}_{2}(10$ mmol. $\left.\left.L^{-1}\right) ;\left(\lambda_{\max }=365 \mathrm{~nm}\right).\right]$

\section{CONCLUSÕES}

Foi possível obter ferritas a partir de resíduos de pilhas, com estrutura espinélio e com alto valor de magnetização, sendo possível sua aplicação como catalisador na degradação de fenol, minimizando, desta forma, os impactos causados pelo descarte das pilhas e de fenol ao meio ambiente. As ferritas sintetizadas a partir de pilhas inutilizadas foram bastante eficientes como catalisadores no processo fotoFenton heterogêneo, removendo $30 \%$ e $90 \%$ de fenol em pH 5,5 e 2,5, respectivamente, em um curto período de tempo (2 h) de irradiação. Além disso, ao final do experimento, este material foi facilmente removido da solução utilizando-se uma barra magnética, evitando a liberação de íons $\mathrm{Fe}^{2+} \mathrm{e}$ $\mathrm{Fe}^{3+}$ ao ambiente. 


\section{AGRADECIMENTOS}

À CAPES, ao CNPq e à FAPEMIG pelo apoio financeiro.

\section{REFERÊNCIAS}

[1] J. C. Afonso, A. P. M. G. Barandas, G. A. P. da Silva, S. G. da Fonseca, Química Nova 26 (2003) 573.

[2] CETESB, Divisão de Toxicologia, Genotoxicidade e Microbiologia Ambiental, disponível em: <http://www. cetesb.sp.gov.br>, acesso em julho de 2010.

[3] R. Andreozzi, V. Caprio, A. Insola, R. Marotta, Catalysis Today 53 (1999) 51.

[4] R. J. Bigdar, Chem. Eng. Prog. 91 (1995) 62.

[5] J. J. Pignatello, Environ. Sci. Techn. 26 (1992) 944.

[6] R. F. P. Nogueira, M. C. Oliveira, W. C. Paterlini, Química Nova 30 (2007) 400.

[7] M. R. A. Silva, A. G. Trovó, R. F. P. Nogueira, J. Photochem. Photobiol. A, Chemistry 191 (2007) 187.

[8] J. J. Pignatello, E. Oliveros, A. Mackay, Critical Rev. Environ. Sci. Techn. 36 (2006) 1.

[9] M. R. A. Silva, M. C. Oliveira, R. F. P. Nogueira, Eclética Química 29 (2004), 19.

[10] M. Noorjahan, V. D. Kumari, M. Subrahmanyam, L.
Panda, Appl. Catal. B: Environ. 57 (2005) 291.

[11] T. Valdés-Sólis, P. Valle-Vigón, S. Álvarez, G. Marbán, A. B. Fuertes, Catal. Comm. 8 (2007) 2037.

[12] F. B. Li, X. Z. Li, C. S. Liu, X. M. Li, T. X. Liu, Ind. Eng. Chem. Res. 46 (2007) 781.

[13] CONAMA, Resolução n. 357, de 17 de março de 2005. Disponível em: <http://www.mma.gov. br/conama/res/ res05/res35705.pdf>.

[14] O. Folin, V. Ciocalteu, J. Biol. Chem. 73 (1927) 627.

[15] M. T. Silva, E. Noulib, N. P. Xekoukoulotakisb, D. Mantzavinosb, Appl. Catal. B: Environ. 73 (2007) 11.

[16] S. J. Stewart, S. J. A. Figueroa, J. M. Ramalho-López, F. G. Requejo, S. G. Marchetti, J. F. Bengoa, R. J. Prado, Phys. Rev. B, 75 (2007) 073408.

[17] R. Gimenes, M. R. Baldissera, M. R. A. da Silva, C. A. da Silveira, D. A. W. Soares, L. A. Perazolli, M. R. da Silva, M. A. Zaghete, Ceram. Int. 38 (2011) 741.

[18] J. Nan, D. Han, M. Cui, M. Yang, L. Pan, J. Hazardous Mater. B133 (2006) 257.

[19] V. J. Silva, A. P. A Diniz, P. T. A. Santos, D. A. Vieira, A. C. F. M. Costa, D. R. Cornejo, L. Gama, Revista Eletrônica Mater. Proc. 1 (2006) 09.

[20] E. Casbeer, V. K. Sharma, X .Z. Li, Separation Purification Techn. 87 (2012) 1.

(Rec. 12/04/2013, Rev. 21/07/2013, Ac. 21/09/2013) 